Rapid Reviews COVID-19

\title{
Reviews of "Inhaled budesonide in the treatment of early COVID- 19 illness: a randomised controlled trial"
}

Ying-Rong $\mathrm{Du}^{1}$, Hai-yan $\mathrm{Fu}^{1}$, Ariel Berlinski ${ }^{2}$

${ }^{1}$ Dali University, sixth affilated hospital, China,

${ }^{2}$ Professor, University of Arkansas for Medical Sciences, Pediatrics

Published on: Apr 26, 2021

DOI: $10.1162 / 2 \mathrm{e} 3983 f 5.3790 \mathrm{e} 6 \mathrm{f9}$

License: Creative Commons Attribution 4.0 International License (CC-BY 4.0). 
To read the original manuscript, click the link above.

Summary of Reviews: This study suggests that budesonide, a corticosteroid used for COPD and asthma treatment, reduces the likelihood of urgent care, ED visit, or hospitalization in patients with early COVID-19; both reviewers found the study findings to be reasonable and potentially reliable.

\title{
Reviewer 1 (Ying-Rong Du, Hai-yan Fu) | |
}

\section{Reviewer 2 (Ariel Berlinski) |}

\author{
RR:C19 Strength of Evidence Scale Key \\ प्रमप = Misleading

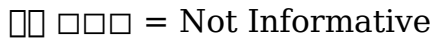 \\ प्र० $\square$ = Potentially Informative \\ प्र०प् = Reliable \\ प्राप्र = Strong
}

To read the reviews, click the links below. 